\title{
Nearest Neighbor Queries in Road Networks
}

\author{
Christian S. Jensen ${ }^{1} \quad$ Jan Koláŕ ${ }^{2} \quad$ Torben Bach Pedersen $^{1} \quad$ Igor Timko \\ ${ }^{1}$ Department of Computer Science, Aalborg University, Denmark \\ ${ }^{2}$ VR Media Lab, Aalborg University, Denmark \\ csj@cs.auc.dk, kolda@vrmedialab.dk, tbp@cs.auc.dk, timko@cs.auc.dk
}

\begin{abstract}
With wireless communications and geo-positioning being widely available, it becomes possible to offer new e-services that provide mobile users with information about other mobile objects. This paper concerns active, ordered k-nearest neighbor queries for query and data objects that are moving in road networks. Such queries may be of use in many services.

Specifically, we present an easily implementable data model that serves well as a foundation for such queries. We also present the design of a prototype system that implements the queries based on the data model. The algorithm used for the nearest neighbor search in the prototype is presented in detail. In addition, the paper reports on results from experiments with the prototype system.
\end{abstract}

\section{Categories and Subject Descriptors}

H.2.2 [Database Management]: Physical Design—access methods; H.2.8 [Database Management]: Database Applications-spatial databases and GIS

\section{General Terms}

Algorithms

\section{Keywords}

Location-based services, road networks, nearest neighbors, query processing

\section{INTRODUCTION}

The proliferation of wireless communications and geo-positioning, along with the general advances in computing technologies enable the delivery of location-aware e-services to mobile users. This means that each service user, e.g., a driver of a car with a built-in computer or an individual equipped with a mobile terminal (phone, PDA, etc.), may receive services that exploit knowledge of the changing locations of all service users.

One single, generic scenario may be envisioned for such services. The moving objects disclose their location information (position,

Permission to make digital or hard copies of all or part of this work for personal or classroom use is granted without fee provided that copies are not made or distributed for profit or commercial advantage and that copies bear this notice and the full citation on the first page. To copy otherwise, to republish, to post on servers or to redistribute to lists, requires prior specific permission and/or a fee.

GIS'03, November 7-8, 2003, New Orleans, Louisiana, USA.

Copyright 2003 ACM 1-58113-730-3/03/0011 ...\$5.00. speed, velocity, etc.) to the services they use, which in turn use this and other information to provide specific functionality. The services function by issuing different types of queries to a database that contains location information, in addition to other content.

In this paper, we consider the support for one such type of query, namely the active ordered $k$-nearest neighbor (NN) query. We assume that the query is issued by a mobile user and concerns other mobile objects; and we assume that all movement is constrained to a road network.

Appropriate representation of the query and data objects and of the road networks in which they move is of essence. Existing data models are inadequate for the query and setting we consider. The relational model resides at a low level of abstraction and offers little customized support for solving the problem. Spatial extensions to SQL (e.g., [10]) as well as modern geographic information systems [8] lack support for dynamic data. Finally, there exist some rich spatio-temporal SQL extensions, perhaps most notably [7]. However, proper implementations of these are still in progress. Moreover, such models generally assume that movement occurs in Euclidean space, not in road networks.

This paper's contribution is on two levels. First, the paper describes a general, abstract framework for NN querying on moving objects in road networks. The framework includes a data model and definitions of abstract functionality needed for NN querying. Second, the paper populates the framework with a set of concrete algorithms and demonstrates the algorithms in a prototype implementation. The framework is general enough to allow other algorithms with other characteristics to be in place of the ones presented here.

Separate elements of the data model capture road networks and data points with continuously changing locations. More specifically, the model encompasses two data representations. The detailed twodimensional (2D) representation captures the geographical coordinates of the roads and moving objects. The more abstract graph representation captures the roads and moving objects in a form that enables efficient NN querying; road distances are used instead of Euclidean distances. Finally, the model may be implemented using the relational model.

The presented algorithms for active, ordered $k$-nearest neighbor querying, and the prototype system implementing the algorithms, employ a client-server architecture that partitions the NN search. First, a preliminary search for a Nearest Neighbor Candidate set (NNC set) is performed on the server. In essence, this search is a best-first search in a graph [9]. Next, the maintenance of the activequery result is done on the client, which recomputes distances between data points in the NNC set and the query point, sorts the distances, and periodically refreshes the NNC set to avoid significant imprecision. The combination of NNC search with the maintenance of an active result provides the user with an up-to-date query result. 
The paper reports on initial experiments with the prototype system.

Previous work on NN search for spatial data differs from the work presented in this paper with respect to the mobility of points and the presence of a road network. Most work on NN search is dedicated to the case where query and data points are embedded in Euclidean space: Solutions exist for the case of static query and data points (e.g., [4]), as do solutions for moving query points (e.g., [13, 14]); active queries for moving query and data points have also been considered [2].

One paper on NN search [12] defines the proximity of moving objects in terms of distances along roads, which we also do. The paper also proposes an algorithm for computing these distances. However, the distances are computed only approximately and the settings are restricted to planar graphs. We compute the distances precisely and assume more general graphs. A recent paper [11] considers NN queries in road network using an approach similar to ours (2D model mapped to a graph model), but considers neither moving objects and query points nor active queries. Yet another paper [5] considers the related, but different problem of computing time-dependent shortest paths using a road network model that is more abstract and thus less precise compared to our approach. Finally, we note that new spatial access methods such as sorted lists [16] or graphs [1] may prove more efficient than existing R-tree-based methods in the mobile setting or with road distances. This is one reason to consider the graph representation in this paper.

The remainder of the paper is structured as follows. Following a description of the problem setting in Section 2, Section 3 present the data model. Next, Section 4 describes the architecture of the prototype system. Section 5 covers the NN search algorithms. Section 6 offers an initial evaluation of our contribution, and Section 7 concludes the paper.

\section{PROBLEM STATEMENT}

Consider a road network with different objects, e.g., taxis, delivery vans, and pedestrians, moving along its roads. All or some of these objects, so-called user objects, or users, may want to continuously monitor the locations of one or more nearest objects, termed nearest neighbors (NNs). This corresponds to an active, ordered $k-N N$ query, an example being "monitor the 3 nearest, free taxis." The resulting objects are sorted in ascending order according to their distance to the query object.

One may perceive a real-world road network, $r n$, as the set of all the locations in 2D Euclidean space covered by the network, termed road locations, $L o c_{r n}$. The locations (points) in the set $L o c_{r n}$ are chosen so that the road network corresponds to a set of curves (see Figure 1) [7]. The widths of roads are ignored, which is reasonable for our applications. We denote the set of all possible road networks by $R N$.

Given the set $L o c_{r n}$, we model a moving object by a data point, $d p_{i}: T \longmapsto L o c_{r n}$, i.e., a function from the time domain $T$ to network locations. We denote the set of all data points by $D P$. Here, we ignore the extents of objects, which is also reasonable for our applications.

Next, we introduce a distance function, dist $t_{r n}: L_{o c} \times{ }_{r n} \times c_{r n}$ $\longmapsto \mathbb{R}_{+}$, where $\mathbb{R}_{+}$is the set of non-negative real numbers. The function calculates the distance between any two network locations, e.g., a data and a query point. The distance function may depend on the characteristics of a road, e.g., its length, quality of surface, maximum speed, etc. However, the distance between two data points does not depend on other characteristics of the argument points than their locations.

We require that the distance function be a metric, which is natural for at least two reasons. First, the distance between two net- work locations must be the length of the shortest (in some particular sense) path between the locations. Second, since we do not distinguish between one- and two-way roads, the distance between two data points must not depend on the direction of their movement. Next, the distance function does not necessarily compute the Euclidean distances. Rather, we expect it to compute distances along the roads, which is the most natural for our applications.
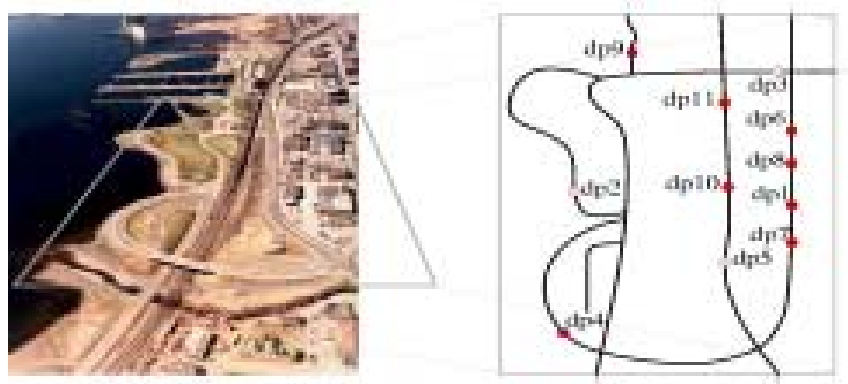

Figure 1: Road Network

EXAMPLE 1. Consider the Arterial Route in Ontario, USA, in Figure 1. We have a road network ont $\in R N$. Furthermore, suppose there are 11 moving objects in the road network, which are taxis, delivery vans, and pedestrians. This means that we have a set of data points $D P_{\text {ont }}=\left\{d p_{i}, i=1, \ldots, 11\right\}$. Finally, assume a road distance function dist $_{\text {ont }}$ is given.

The active, ordered $k$-NN query takes three parameters. First, the query point, $q p \in D P$, represents the user object that has issued the query. A query point is a data point. Second, the number of NNs, $k \in \mathbb{N}$, where $\mathbb{N}$ is the natural numbers, indicates the number of NNs to be monitored. Third, the data point property predicate, prop : $T \times D P \longmapsto B o o l$, describes properties that qualifying objects must satisfy. The properties of real-world objects are often dynamic. For example, a free taxi picks up a passenger, becoming occupied. For this reason, the value of a data point property predicate depends on time.

EXAMPLE 2. Continuing Example 1, we formulate the query "monitor the 3 nearest, free taxis," issued by a pedestrian represented by $d p_{11}$. This query takes the following parameters: a query point $q p=d p_{11}$, a number of NNs $k=3$, and a data point property predicate $\operatorname{prop}_{\text {free }}$, defined as $\operatorname{prop}_{\text {free }}(t, d p) \Leftrightarrow$ " $d p$ is a free taxi at time point $t$."

An active, ordered $k$-NN query issued at time $t$ returns a list of $k$ NNs $R L(t)$ that satisfies the property predicate. The NNs are ordered by distance in ascending order, and the list contains neither the query point, nor any duplicates. The result of the query remains up-to-date from when the query is issued and until it is discontinued.

\section{DATA MODEL}

The model of road networks and data points presented next aim to support a system that provides users with active NN query functionality. Positioning units enable the users to report their positions, and the query results are displayed in their geographical contexts. The data model includes a two-dimensional representation of road networks and data points that supports user-interaction tasks, e.g., visualizing results in their geographical context, and a graph representation that supports the NN querying. 


\subsection{Two-Dimensional Representation}

The two-dimensional (2D) representation captures data in its concrete, geographical form, i.e., with a direct link to a coordinate system. To be precise, the key elements of the representation, i.e., vertices, lines, and data points, are described by points or sets of points in the Euclidean plane.

Two-Dimensional Road Network Representation. In Section 2, we presented a simple model of a real-world road network. We now use this model to specify the link between the 2D representation and the real world.

Given a road network $r n$, the corresponding $2 D$ road network is a three-tuple $2 d r n=\left(V_{2 d r n}, V C_{2 d r n}, L_{2 d r n}\right)$. We denote the set of all such road networks by $2 D R N$.

The finite set of vertices, $V_{2 d r n}=\left\{v_{1}, \ldots, v_{n}\right\}$, is partitioned into the sets $V_{2 d r n}^{t}, V_{2 d r n}^{i s}$, and $V_{2 d r n}^{i m}$. A one-to-one vertex coordinate function $V C_{2 d r n}: V_{2 d r n} \longmapsto \mathbb{R}^{2}$, maps each vertex $v$ to its point location in the Euclidean plane. The number, density, and placement of vertices in $2 d r n$ is chosen so that any location in $L o c_{r n}$ is represented with sufficient accuracy in $C_{2 d r n}$, which is crucial for pinpointing a data point in the $2 \mathrm{D}$ representation (see below).

The set of lines, $L_{2 d r n} \subseteq V_{2 d_{r n}} \times V_{2 d r n}$, approximates the curves given by the locations $\operatorname{Loc}_{r n}$. Each line $\left(v_{i}, v_{j}\right) \in L_{2 d r n}$ has a start vertex, $v_{i}$, and an end vertex, $v_{j}$, allowing indication of the direction of movement of data points (see below).

Having defined lines, we can identify three types of vertices. A terminal vertex, $v_{t} \in V_{2 d r n}^{t}$, is adjacent to only one line. An intersection vertex, $v_{i s} \in V_{2 d r n}^{i s}$, is adjacent to at least three lines. Finally, an intermediate vertex, $v_{i m} \in V_{2 d r n}^{i m}$, is a vertex that is neither terminal nor intersection.

For data point distance computation, we capture a few more road network characteristics. Given a line $\left(v_{i}, v_{j}\right)$, the line length function, LLen $2 d r n: L_{2 d r n} \longmapsto \mathbb{R}_{+} \backslash\{0\}$, gives the Euclidean distance between $V C_{2 d r n}\left(v_{i}\right)$ and $V C_{2 d r n}\left(v_{j}\right)$, and the line maximum speed function, $\operatorname{LMax}_{2 d r n}: L_{2 d r n} \longmapsto \mathbb{R}_{+} \backslash\{0\}$, indicates the speed limit for objects moving along the line.

Two-Dimensional Data Point Representation. According to Section 2, a data point $d p \in D P$ is, in essence, a function that captures the trajectory of an object for its lifespan. Such a representation is inadequate for capturing inherently discrete data obtained from the positioning units attached to the moving objects. Thus, for the $2 \mathrm{D}$ representation, we redefine $D P$ as a set of data points without any characteristics, i.e., a set of data point "surrogate ids."

We define the following data point characteristics (DPCs) that model the data obtained from the positioning unit. First, the $2 D$ data point reference time function, $T_{2 d r n}: D P \longmapsto T$, provides the reference time of a data point, which is the time of the last reported measurement for the data point. Second, the data point coordinate function, $C_{2 d r n}: D P \longmapsto C_{2 d r n}$, gives the $2 \mathrm{D}$ coordinates for a data point at its reference time. Third, the data point speed function, $S_{2 d r n}: D P \longmapsto \mathbb{R}$, gives the speed of a data point at its reference time.

EXAMPLE 3. Continuing the earlier examples, we consider the Arterial Route road network ont $\in R N$. In the $2 \mathrm{D}$ representation, network ont is a three-tuple 2 dont $=\left(V_{2 \text { dont }}, V C_{2 \text { dont }}, L_{2 \text { dont }}\right)$. Specifically, the part of Water Street depicted in Figure 2 can be modeled by three lines $l_{1}, l_{2}, l_{3} \in L_{2 d o n t}$. These are shown in the first table below, which also contains the length and maximum speed attributes of the street. The location attributes of the street are given by the $2 \mathrm{D}$ coordinates shown in the second table below. Next, there are two moving data points in Figure $2, d p_{1}$ and $d p_{2}$. In the $2 \mathrm{D}$ representation, a data point $d p$ is given at the last reference time $T_{2 \text { dont }}(d p)$ by its coordinates $C_{2 d o n t}(d p)_{x}$ and $C_{2 d o n t}(d p)_{y}$, and its speed $S_{2 d o n t}(d p)$. A positive speed value indicates movement from the start vertex to the end vertex, and a negative value indicates the opposite. The third table below summarizes the characteristic of the two data points.

\begin{tabular}{|c|c|c|c|c|}
\hline $\begin{array}{l}\text { Line } \\
l\end{array}$ & $\begin{array}{l}\text { Start Vertex } \\
v_{i}\end{array}$ & $\begin{array}{l}\text { End Vertex } \\
v_{j} \\
\end{array}$ & $\begin{array}{l}\text { Length } \\
\operatorname{LLen}(l) \\
\end{array}$ & $\begin{array}{l}\text { Max. Speed } \\
L M a x S(l) \\
\end{array}$ \\
\hline$\overline{\overline{l_{1}}}$ & " & $\overline{c 681}$ & "350 & " \\
\hline$l_{2}$ & 681 & 682 & 84 & 16.7 \\
\hline$l_{3}$ & 682 & 624 & 90 & 16.7 \\
\hline & $\begin{array}{l}\text { Vertex } \\
v\end{array}$ & $\begin{array}{l}\text { X-coordinate } \\
V C(v)_{x}\end{array}$ & $\begin{array}{l}\text { Y-coordi } \\
V C(v)_{y}\end{array}$ & \\
\hline & 8680 & ' & "17825 & \\
\hline & 681 & 11057 & 17825 & \\
\hline & 682 & 11115 & 17799 & \\
\hline & 624 & 11241 & 17738 & \\
\hline $\begin{array}{l}\text { Point } \\
d p\end{array}$ & $\begin{array}{l}\text { Ref. Time } \\
T(d p)\end{array}$ & $\begin{array}{l}\text { X-coord. } \\
C(d p)_{x}\end{array}$ & $\begin{array}{l}\text { Y-coord. } \\
C(d p)_{y}\end{array}$ & $\begin{array}{l}\text { Speed } \\
S(d p) \\
\end{array}$ \\
\hline$d p_{1}$ & 9114877 & 111033 & 17825 & -15.52 \\
\hline$d p_{2}$ & 9114877 & 11033 & 17825 & 12.01 \\
\hline
\end{tabular}

\subsection{Graph Representation}

We define the graph representations of a road network and data points, and we relate them to the $2 \mathrm{D}$ representation.

Graph Road Network Representation. Assume a real-world road network $r n$, modeled as described in Section 2. In the 2D representation, a sequence of lines approximates the shape of the real road network. In the graph representation, such sequences may be mapped to a single edge. The ideas are to (i) not consider geographical location, while (ii) still preserving the topology of the road network. Nevertheless, the graph representation must still refer back to the $2 \mathrm{D}$ representation. This is necessary for the visualization of $\mathrm{NN}$ query results.

Formally, given a real-world road network $r n \in R N$, the graph road network is a two-tuple grn $=\left(N_{g r n}, E_{\text {grn }}\right)$. We denote the set of all such road networks by GRN. The finite set of nodes, $N_{\text {grn }}=$ $\left\{n_{1}, \ldots, n_{n}\right\}$, is partitioned into a set of transformed nodes, $N_{g r n}^{t f}$, and a set of additional nodes, $N_{g r n}^{a}$. The set of directed edges is $E_{\text {grn }} \subseteq N_{\text {grn }} \times N_{\text {grn }} \times I D$, where $I D$ is the domain of identifiers. The edge from a start node, $n_{i}$, to an end node, $n_{j}$, is denoted by $E d g e\left(n_{i}, n_{j}, i d\right)$. By using directed edges, we obtain a means of indicating the movement directions of data points.

Next, we transform a 2D road network, denoted $2 d r n=\left(V_{2 d r n}\right.$, $\left.L_{2 d r n}, V C_{2 d r n}\right)$, to its corresponding graph representation, $g r n=$ $\left(N_{g r n}, E_{g r n}\right)$. First, we omit $V C_{2 d r n}$ because the graph representation has no means of geographical referencing. Second, each path, i.e., line or non-closed sequence of lines, for which (i) the end vertices are intersection or terminal vertices and (ii) all other vertices are intermediate vertices, is collapsed into a single edge. Because we may obtain edges with an identical pair of end nodes, e.g., the two edges between nodes 600 and 604 in Figure 2, we assign an $i d$ to each edge. Consequently, each intersection and terminal vertex results in one transformed node. Finally, each cycle, i.e., closed sequence of lines for which all vertices but one are intermediate, is transformed into a pair of edges and one additional node. This avoids loops, i.e., edges that start and end at the same node, which ensures that we are always able to unambiguously specify the movement direction of a data point (see below). We note that while the $2 \mathrm{D}$ model and the transformation from the 2D to the graph model 


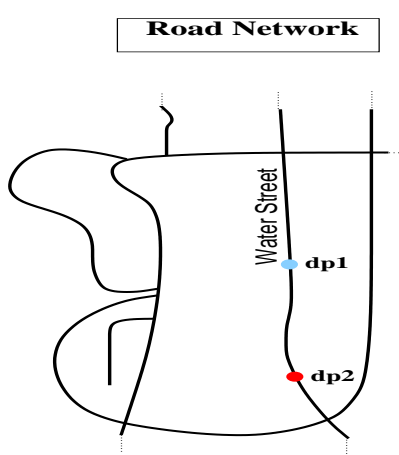

Arterial Route region ONTARIO, USA

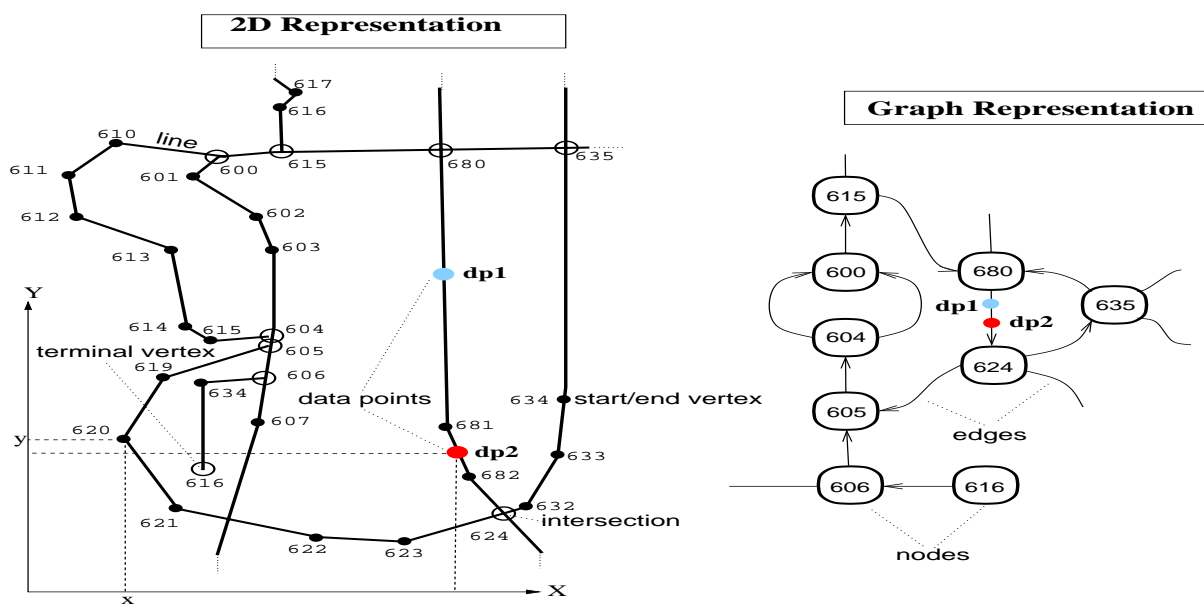

Figure 2: Two-Dimensional and Graph Representations of Road Network

does not consider directional attributes and traffic regulations such as turn restrictions (for simplicity), the underlying graph model in which all computation takes place is not affected by these. Thus, all that is needed to support such aspects is more elaborate transformations that generate more accurate and complex graphs. A number of such transformations are available in the literature.

To maximally simplify proximity computation in graphs, we only introduce one edge characteristic, namely the edge weight function, $E W_{g r n}: E_{\text {grn }} \longmapsto R_{+} \backslash\{0\}$. Weights are computed differently for edges obtained from transformations of different types, but the idea remains the same: the weight of an edge reflects the minimum time needed to travel from the start vertex to the end vertex of the corresponding 2D path. For an edge obtained by transforming a path, the travel time is the sum of the times obtained for each line of the path. For a pair of edges obtained by transforming a cycle, the travel time is half the time needed to cover the whole cycle, i.e., the additional node is inserted at the middle of the loop.

Graph Data Point Representation. A graph data point is analogous to a $2 \mathrm{D}$ data point, so set $D P$ is common for both representations. However, we also introduce a function for tracking a data point's position in the graph representation.

A data point graph reference time function, $T_{g r n}: D P \longmapsto T$, is introduced. The data point edge function, $E_{g r n}: D P \longmapsto E_{g r n}$, returns the current edge of an argument data point. The data point initial position function, IPos $s_{\text {rn }}: D P \longmapsto \mathbb{R}_{+}$, returns the distance from the start of the current edge of the argument data point. The combination of values of the latter two functions define the position of a data point in the graph at the reference time. Finally, the data point speed function, $S_{\text {grn }}: D P \longmapsto \mathbb{R}$, is analogous to the speed function in 2D.

For NN search, we need to know the positions of data points in the graph representation not only at some reference time, but at arbitrary times. For this purpose, we introduce a positioning function, Pos grn $: T \times D P \longmapsto \mathbb{R}_{+}$, that returns the distance of a moving point from the start node of its current edge, i.e., $\operatorname{Pos}_{g r n}(t, d p)=$ $=I P \operatorname{sos}_{g r n}(d p)+S_{g r n}(d p) \times\left(t-T_{g r n}(d p)\right)$, with the condition that $\operatorname{Pos}_{g r n}(t, d p) \in\left[0 ; E W_{g r n}\left(E_{g r n}(d p)\right)\right]$.

Also, $I P \operatorname{Pos}_{g r n}(d p), T_{g r n}(d p)$, and $S_{g r n}(d p)$ denote the most recent values for $d p$ in the graph. Thus $\operatorname{Pos}_{g r n}(t, d p)$ returns $d p$ 's position on the edge at the current time $t$, or, more precisely, an approximation of $d p$ 's real position with the assumption that the motion is at constant speed $S_{g r n}(d p)$ since the last update of the data.
In order to both simplify the definition and mimimize the approximation error of the positioning function, the values of the function are limited to positions on the current edge.

As the graph representation of a road network is obtained from its 2D representation, there is a dependency among the corresponding data point functions. For example, the speed function in the graph representation is defined using the $2 \mathrm{D}$ speed function. In this paper, we limit ourselves to discussing the initial position function, which is in essence a $2 \mathrm{D}$-to-graph transformation of data point locations at a reference time.

Ad for edge weights, initial data point positions are computed differently, depending on the type of transformation that led to the current edge. However, the idea again remains the same: the distance between the data point and the start node of the current edge is derived from the corresponding distance in the 2D road network. Absolute 2D distances are transformed to ones that relative to the lengths of the paths/cycles under consideration. In turn, the relative distances are transformed to absolute ones in the graph road network. This is done by multiplying the former distances by the weights of the current edges, for edges corresponding to paths, or by twice the weights of the edges, for edges corresponding to half cycles.

EXAMPLE 4. Continuing Example 3, the graph representation of the considered part of Water Street is modeled by one edge $e_{1}$ with nodes 680 and 624 . As there is no geographic referencing, the three tables from Example 3 collapse into the following table:

\begin{tabular}{|c|c|c|c|}
\hline $\begin{array}{c}\text { Edge } \\
e\end{array}$ & $\begin{array}{c}\text { Start Node } \\
n_{i}\end{array}$ & $\begin{array}{c}\text { End Node } \\
n_{j}\end{array}$ & $\begin{array}{c}\text { Edge Weight } \\
E W(e)\end{array}$ \\
\hline \hline$e_{1}$ & 680 & 624 & 32.3 \\
\hline
\end{tabular}

The two moving data points have the same reference time as in the $2 \mathrm{D}$ representation. However, the coordinates of a data point $d p$ are replaced by the current edge $E_{\text {gont }}(d p)$ and initial position $I_{\text {IPos }}$ gont $(d p)$. The current position of $d p$ on an edge is given by the positioning function Posgont. Finally, the speed $S_{\text {gont }}(d p)$ is analogous to the speed $S_{2 d o n t}(d p)$. The following table presents the characteristics of the two data points in the graph representation:

\begin{tabular}{|c|c|c|c|c|}
\hline Point & $\begin{array}{c}\text { Ref. Time } \\
T(d p)\end{array}$ & $\begin{array}{c}\text { Edge } \\
E(d p)\end{array}$ & $\begin{array}{c}\text { Init. Pos. } \\
I P o s(d p)\end{array}$ & $\begin{array}{c}\text { Speed } \\
S(d p)\end{array}$ \\
\hline \hline$d p_{1}$ & 9114877 & $e_{1}$ & 10.3 & -0.929 \\
\hline$d p_{2}$ & 9114877 & $e_{1}$ & 10.3 & 0.719 \\
\hline
\end{tabular}




\section{SYSTEM ARCHITECTURE}

We proceed to design a software system that implements $\mathrm{NN}$ queries. The system satisfies the requirements stated in Section 2, and it exploits the data model described in Section 3.

A client-server architecture is natural for the problem considered: moving users work directly with the client software installed on their mobile device; and the server assists the clients in providing users with the NN query results. The architecture allows to us to distribute data storage and computation between the server and the clients in an implementable manner. The resource-heavy tasks, e.g., managing the database with DPCs of all data points, are assigned to the server, while relatively simple tasks specific to an individual user are left to the clients.

Client-Side Tasks. Clients correspond to the moving objects in the system. A client can play the role of a query point or data point, or both. We consider the tasks performed by query points. The main task of a client that is a data point only is to send updates of its characteristics to the server.

The request for NNC set is the starting point of NN computation. When a user asks the client for specific NNs, the client first requests an appropriate NNC (nearest neighbor candidate) set from the server. This allows the client to work with only currently relevant data, i.e., a small subset of the data points instead of the whole database. This is important for client side computational efficiency. Moreover, for some time, the NNC set allows the client to work offline, i.e., without any additional data from the server, reducing the communication network load.

Having received the $\mathrm{NNC}$ set, the client starts maintaining the active query result (see Section 2). This task includes three main subtasks: NNC validation, distance computation, and NNC sort.

The task of NNC validation controls when to refresh the NNC set from the server. In the system, the NNC set is built at one point in time (see Section 5.2) and contains the same data points until the next request for the NNC set. The client therefore estimates the imprecision of the NNC set to decide when to next request the NNC set. This estimate is made using the expiration number, which is the maximum number of data points from the NNC set that are allowed to be beyond a certain distance limit from the query point. By using different values of the expiration number, we can control the tradeoff between the imprecision of the NN result and the communication network and server loads. An algorithm for NNC validation is presented in Section 5.4.

Distance computation continuously returns the distance between the query point and any data point in the NNC set, which is essential for sorting the data points in the NNC set. The distance is expressed in terms of the graph representation. A distance computation algorithm is introduced in Section 5.3.

In any location-based service, a major task is client positioning. Each client obtains its position from an attached positioning unit and stores the position into the DPC in the 2D representation (see Section 3.1). Since this so-called client DPC reflects the client's exact location, we consider the location given by the client DPC as correct (with respect to the system). The client uses its DPC for maintaining the active result and for DPC update, which is described next.

The client sends its DPC to the server, i.e., performs DPC update. The resulting server DPC attributes are kept in a database of moving points. The main issue of DPC update is how often a client should send its DPC to the server. Frequent updates increase the communication network and server loads; and infrequent updates introduce significant deviations between the client and server DPCs, which can render the database unusable for determining the positions of

the moving points.

DPC update exploits the dead-reckoning (DR) policy of [15]: an update occurs whenever the deviation of the location given by server DPC from the corresponding location given by client DPC exceeds a given threshold. We use a (in graph travel-time terms) fixed threshold, i.e., the inaccuracy relative to the speed of the objects is constant, but the absolute inaccuracy (measured in real-world distance) grows with the speed limit (see the edge weight definition in Section $3.2)$.

Server-Side Tasks. For each client NNC request, the server builds the NNC set and sends it back. The NNC search scans the road network in the graph representation in order to gather a given number of data points that fulfill the client query. The scan propagates from the current location of the query point along all possible paths until the required number of data points is found or until the entire graph is scanned. In addition, the server keeps track of all currently used NNC sets and the clients that are using them. An algorithm for NNC search is presented in Section 5.2.

The most frequent server-side data management task is the management of the database of moving points. Even without any submitted NNC set requests, the server has to manage data on the movement of all data points. For each data point, the server updates the attributes of the DPCs (see Section 3.2) in the database of moving points. When processing a DPC update, the server monitors the $N N C$ sets, i.e., checks whether the corresponding data point is included in any of the currently used NNC sets. If so, the server sends the new server DPC to the appropriate client(s), even though the client has not requested a new NNC set.

\section{NEAREST NEIGHBOR ALGORITHMS}

This section is dedicated to the details of our NN search algorithm. The top-level algorithm is presented first. Then the constituent algorithms for nearest neighbor candidate search, distance computation, and nearest neighbor candidate validation are presented in turn.

\subsection{Top-Level Algorithm}

The NN search algorithm is the key function of the software system presented in Section 4. Three system tasks, i.e., NNC requests, active result maintenance, and NNC search, use the algorithm. The algorithm is seen below.

(1)

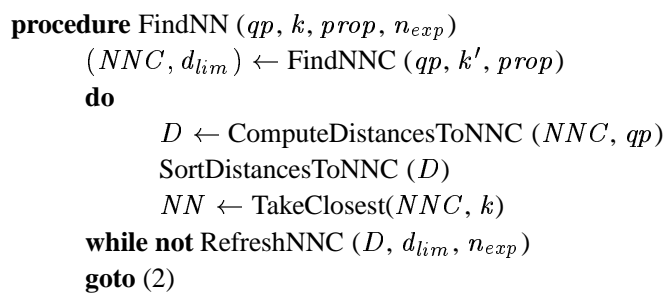

The procedure FindNN computes the active, ordered $k$-NN query as stated in Section 2. In particular, the first three parameters of the procedure correspond to the active, ordered $k$-NN query parameters (see Section 2). The last parameter $n_{\text {exp }}$ is the expiration number discussed in Section 4. The current result of the procedure, i.e., the current NNs, is stored in $N N$ (line 6). Note also that the call of the procedure TakeClosest returns up to $k$ closest elements of the $N N C$ set.

The links between the mentioned system tasks and the sub-procedures are as follows. First, the call of the FindNNC procedure in line 3 corresponds to the task of NNC requests. Second, the FindNNC procedure itself is used by the task of NNC search. Finally, 
the whole procedure FindNN may be viewed as the task of active result maintenance, with the subtasks of distance computation (procedure ComputeDistancesToNNC in line 4), NNC sort (procedure SortDistancesToNNC in line 6), and of NNC validation (predicate RefreshNNC in line 7). In Sections 5.2-5.4, we discuss the subprocedures in detail.

Note that in procedure FindNN, we run the sub-procedures sequentially, while in the real system, they may run in parallel, e.g. the FindNNC and ComputeDistancesToNNC procedures. In addition, procedure FindNN does not reflect some auxiliary tasks run in the real system, e.g., the NNC monitoring. Finally, procedure FindNN presents the client-side view of the algorithm only, i.e., all the procedures would be executed on the client. These abstractions simplify the presentation of the algorithm without introducing any ambiguity. For discussion of the algorithm in the context of the software system, see Section 4.

\subsection{Nearest Neighbor Candidate Search}

Section 4 describes the purpose of this server-side task. Here, we describe only concepts regarding the algorithm.

In the procedure FindNN, the algorithm for NNC search is represented by the procedure FindNNC. The procedure takes three parameters. The parameter $q p$ identifies the query point, for which the $\mathrm{NN}$ search is performed. The parameter prop state the properties of the data points that we are interested in during the NNC search. These parameters are simply passed from procedure FindNN to procedure FindNNC. The parameter $k^{\prime}$ is the number of data points to include in the NNC set. Naturally, $k^{\prime}$ is greater than or equal to the number of NNs $k$. The procedure returns the pair of the NNC set and the distance limit $\left(N N C, d_{\text {lim }}\right)$.

Due to space constraints, we do not present the pseudocode for the algorithm. In essence, a traditional best-first search [9], extended with the "reading" of data points from edges, is performed. The search starts at the current query point and extends outwards through the road network. The NNC set found contains the true, i.e., not approximate, NNs. The purpose of an NNC set that is larger than the desired $k \mathrm{NNs}$ is to make the NNC set more stable to change over time, and thus make the maintenance of the active, ordered query result perform better.

\subsection{Distance Computation}

The active result maintenance task uses the distance computation algorithm (see Section 4). Although that task uses the algorithm for computation of the distance between the query point and a data point from an NNC set, it can be applied to two arbitrary data points. Briefly, the algorithm computes the length of the shortest path between two data points. The difficulty is that with continuously moving data points and alternative paths between the points present, we must continuously recompute the shortest path.

In order to present the algorithm, we introduce several functions. Given a data point $d p$ and its current edge $e$ with start node $a$ and end node $b$, its distance residuals with signatures $r_{a}^{d p}: T \longmapsto \mathbb{R}_{+}$and $r_{b}^{d p}: T \longmapsto \mathbb{R}_{+}$, are defined as $r_{a}^{d p}(t)=\operatorname{Pos}_{g r n}(t, d p)$ and $r_{b}^{d p}(t)=$ $E W(e)-r_{a}^{d p}(t)$. In other words, for a given data point, the functions return its distance to the nodes adjacent to the current edge along that edge.

Let $|a x|$ denote the length of the shortest path between nodes $a$ and $x$. Given two data points $d p_{1}$ and $d p_{2}$ and their current edges $e_{1}=E d g e\left(a, b, i d_{1}\right)$ and $e_{2}=E d g e\left(x, y, i d_{2}\right)$, the distance function, $d: T \times D P \times D P \longmapsto \mathbb{R}_{+}$, is defined as follows:

$$
d\left(t, d p_{1}, d p_{2}\right)= \begin{cases}\min \left(d_{a x}, d_{b x}, d_{a y}, d_{b y}\right), & \text { if } e_{1} \neq e_{2} \\ \min \left(d_{d i r}, d_{\text {out }}\right), & \text { if } e_{1}=e_{2}\end{cases}
$$

where

$d_{a y}=|a y|+r_{y}^{d p_{2}}(t)+r_{a}^{d p_{1}}(t), d_{a x}=|a x|+r_{x}^{d p_{2}}(t)+r_{a}^{d p_{1}}(t)$, $d_{b x}=|b x|+r_{x}^{d p_{2}}(t)+r_{b}^{d p_{1}}(t), d_{b y}=|b y|+r_{y}^{d p_{2}}(t)+r_{b}^{d p_{1}}(t)$, $d_{d i r}=\left|r_{x}^{d p_{2}}(t)-r_{a}^{d p_{1}}(t)\right|, d_{\text {out }}=|a b|+\left(E W\left(e_{2}\right)-d_{\text {dir }}\right)$.

Recall that we require that the distance function be a metric. The function presented in this section satisfies this requirement.

EXAMPLE 5. Figure 3 shows a graph road network with edge weights and two data points, $d p_{1}$ and $d p_{2}$. According to the distance function definition, at the time $t$ depicted in the figure, the distance between the moving points $d\left(t, d p_{1}, d p_{2}\right)$ is 11 .

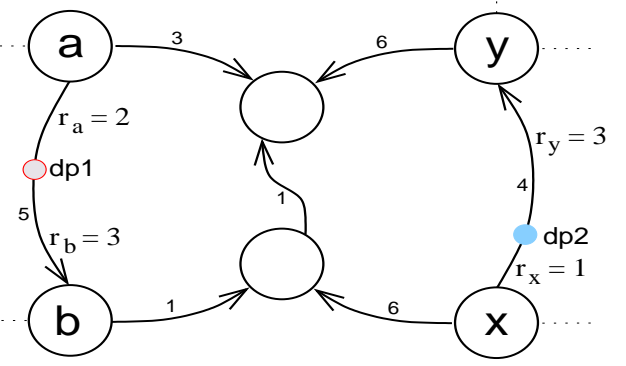

Figure 3: Distance between Two Moving Data Points

Our algorithm uses the shortest distances between nodes. We argue that in the mobile setting it may be too expensive to compute these distances at run-time using an existing shortest path algorithm, e.g., Dijkstra's algorithm [6]. Instead, we choose to pre-calculate the shortest distances between any pair of nodes in a so-called database of distances. This pre-computation allows us to compute the shortest distances between data points as the minimum of only four quickly computable candidate distances.

In procedure FindNN from Section 5.1, the algorithm for distance computation is represented by the procedure ComputeDistancesToNNC. The procedure takes two parameters. The first parameter is an NNC set $N N C$ returned by procedure FindNNC. The second parameter $q p$ is a query point, for which the $\mathrm{NN}$ search is performed. The procedure returns the set of distances $D$ between the data points in $N N C$ and the query point.

(1) procedure ComputeDistancesToNNC $(N N C, q p)$ 


\subsection{Nearest Neighbor Candidate Validation}

While discussing the task of active result maintenance in Section 4, we mentioned the subtask of NNC validation. In the procedure FindNN from Section 5.1, the subtask is implemented by the predicate RefreshNNC. Suppose the number of data points in the NNC set returned by the procedure FindNNC is $k^{\prime}$. Next, suppose the same procedure returned the distance limit $d_{\text {lim }}$. Furthermore, let $n_{\text {exp }} \in 1, \ldots, k^{\prime}$ be the expiration number. Finally, $D$ is the list of distances between a given query point $q p$ and the data points in $N N C$ returned by the procedure ComputeDistancesToNNC. NNC validation is based on the following idea: if "too many" nearest neighbor candidates, i.e., greater than or equal to $n_{\text {exp }}$, are "too far" away from the query point $q p$, then we must refresh the $N N C$ set. A data point $d p$, being too far away means that the point is further from the query point $q p$ than distance limit $d_{\text {lim }}$. Thus, formally the predicate is defined as RefreshNNC $\left(D, d_{\text {lim }}, n_{\text {exp }}\right) \Leftrightarrow$ CountGreater $\left(D, d_{\text {lim }}\right) \geq n_{\text {exp }}$, where CountGreater $(D, d)$ is the number of elements greater than or equal to $d \in \mathbb{R}$ in the list of real numbers $D$.

\section{EVALUATION}

We first discuss pertinent aspects of the data model from Section 3 and the algorithms from Section 5. Next, we briefly describe experiments with the prototype from Section 4, covering settings and some findings. Due to space constraints, only the most significant experiments are described.

\subsection{Discussion}

Our data model includes a 2D and a graph representation of road networks. The 2D-to-graph transformation of a network usually results in a more compact data structure. For example, the road network in the 2D representation for the city of Oldenburg, Germany, consists of 6105 vertices and 7035 lines, while the corresponding graph representation has 2945 nodes and 3875 edges. This encourages us to use the $2 \mathrm{D}$ representation only in tasks where a direct link with the real world is necessary, e.g., visualization of query results. In turn, the graph representation is used for queries, e.g., NN queries, that rely on network topology, but do not rely on geographical coordinates.

By pre-computing the shortest distances between road network nodes in the graph representation, we reduce distance computation to only a few arithmetic operations. Obviously, there is a trade-off between time and space complexity, i.e., the database of distances requires significant amount of storage space. For example, with 2 bytes for each node ID and 2 bytes for distances between nodes, the database for Oldenburg occupies $25 \mathrm{MB}$. However, this amount of space is already available on some mobile devices at the present time, and will be increasingly available on future devices. Note that the 2D-to-graph transformation of road networks reduces size of the database considerably. For example, for Oldenburg the size of the database of distances between vertices is $107 \mathrm{MB}$ for the 2D representation, which is around four times greater. We note that the generality of our framework will allow the use of other methods of distance computation that use less space at the cost of increased computation time. For example, one could imagine a method based on, e.g., a combination of distance computation from scratch and dynamic programming/caching, allowing a tradeoff between space use and computation time.

With our graph representation, classical algorithms for graphs may be reused. For example, our algorithm for NNC search is basically a traditional best-first search. In the algorithm for NNC validation, the notion of expiration number is a simple and effective means of controlling the imprecision of the NN search.

\subsection{Experiments}

In our experiments, we compared two possible graph representations of the road network of the city of Oldenburg. In the first, so-called transformed, graph (TG), nodes and edges are derived exactly as described in Section 3.2. In the second, so-called original, graph (OG), each node and edge correspond to exactly one vertex and one line in the $2 \mathrm{D}$ representation, respectively.

We created our experimental data using a modified version of Brinkhoff's moving object generator [3]. There is one data set for each combination of a) the number of data points $(100,500,1000$, and 5000), and b) the graph (OG and TG). Each data set consists of 1000 simulated GPS measurements for each data point, performed with $1 \mathrm{sec}$ intervals. The starting nodes of the data points were chosen randomly, and the maximum speed of each data point was randomly chosen between $180 \mathrm{~km} / \mathrm{h}, 144 \mathrm{~km} / \mathrm{h}, 90 \mathrm{~km} / \mathrm{h}, 72 \mathrm{~km} / \mathrm{h}, 18$ $\mathrm{km} / \mathrm{h}$, and $9 \mathrm{~km} / \mathrm{h}$ (note that all values of speed and distance in this section are only approximate, due to the data generator).

We ran experiments for certain combinations of the following eight parameter values: 1) the number of data points, 2) the graph, 3 ) the number $k$ of NNs, 4) the maximum query point speed, 5) the number $k^{\prime}$ of NNCs, 6) the expiration number, 7) the update threshold, and 8) the DPC update policy. The experiments were performed in two series. Each series had a particular goal, fixing or varying the mentioned parameters accordingly.

In the first series, a query point issues a given query, with the only query parameter of interest being the number $k$ of NNs. We varied the number of data points $(100,500,1000$, and 5000), the graph (OG and TG), the number $k$ of NNs $(2,10$, and 100), the maximum query point speed $(9 \mathrm{~km} / \mathrm{h}, 90 \mathrm{~km} / \mathrm{h}$, and $180 \mathrm{~km} / \mathrm{h})$, the number $k^{\prime}$ of NNCs $(k+1,1.5 \times k$, and $2 \times k)$, and the expiration number $n_{\text {exp }}\left(1, \frac{1}{2} \times\left(k^{\prime}-k\right)\right.$, and $\left.k^{\prime}-k\right)$. The fixed parameters were the DPC update policy (exactly as described in Section 4) and the update threshold (100 edge weight units, which corresponds to 15 $\mathrm{m}$ for a maximum speed of $54 \mathrm{~km} / \mathrm{h}$ ).

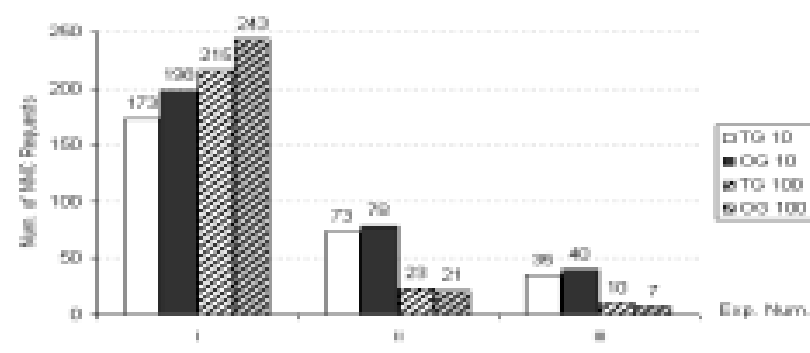

Figure 4: NNC Requests Depending on Expiration Number

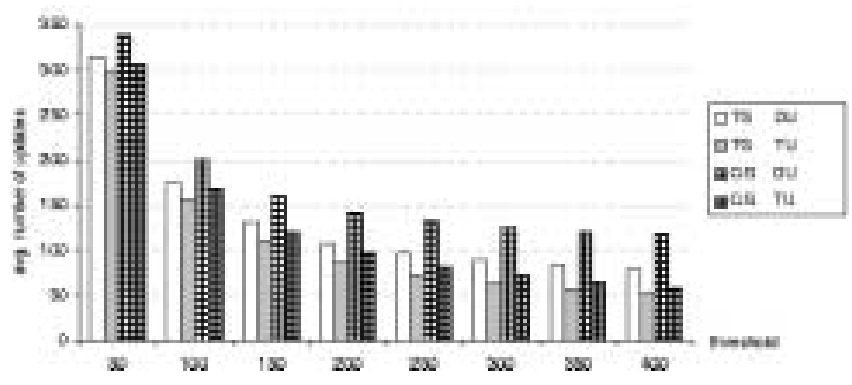

Figure 5: DPC Updates Depending on Threshold

Figure 4 presents the dependency of the average number of NNC requests, made during one experiment by the query point, on the ex- 
piration number $n_{\text {exp }}$ (labels of the $\mathrm{X}$ axis denote cases of (i) 1, (ii) $\frac{1}{2} \times\left(k^{\prime}-k\right)$, or (iii) $k^{\prime}-k$ ), the number $k$ of NNs and the graph (legend items denote a particular combination, e.g., "TG 100" denotes TG with $k=100$ ). In general, this reflects the communucation network and server load. The data is drawn from the subseries of the first series of experiments where we used 1000 data points, a maximum query point speed of $90 \mathrm{~km} / \mathrm{h}$, and $k^{\prime}=1.5 \times k$.

First of all, it is reasonable to assume that due to the difference in edge length, each NNC search is more efficient in the TG than in the OG. Moreover, the figure shows that with $k=10$ the TG yields less NNC requests than the OG. This is because edges in the OG are shorter than in the TG. For this reason, according to our DPC update policy, data points in the OG are more frequently kept at their current edges' nodes before transiting to new edges than in the TG, increasing the chance for an individual NNC to expire. Moreover, the difference in the number of requests is most significant (15\%) with $n_{e x p}=1$ and decreases with bigger expiration numbers, when the increased chances of an individual NNC to expire at arbitrary point in time do not influence the validity of the whole NNC set so much.

We note that with growing number of NNs, the expiration number grows accordingly. For this reason, with $k=100$, the TG is only more efficient than the $\mathrm{OG}$ in terms of $\mathrm{NNC}$ requests in the case where $n=1,(10 \%)$. Rather, with larger expiration number values and $k=100$, the TG demonstrates worse performance than the OG. However, in our targeted scenario, $k$ will almost never be more than 10 or so, making the TG the best choice.

In the second series of experiments, we compare two policies of updates after passing a node. According to the first policy, the socalled direct DPC update, or DU, the DPC update occurs immediately. The second policy, the so-called threshold DPC update, or TU, is exactly as presented in Section 4. We varied the graph (TG and OG), the threshold (50-400 edge weight units with the step of 50 units), and the DPC update policy (TU and DU). We chose to set the lowest threshold value to 50 units, which with a maximum speed of $54 \mathrm{~km} / \mathrm{h}$ corresponds to $7.5 \mathrm{~m}$, a reasonable minimum for city travel at this speed. We set the number of data points to 1000 , the maximum query point speed to $9 \mathrm{~km} / \mathrm{h}, k=1, k^{\prime}=1$, and $n_{\text {exp }}=1$.

Figure 5 shows how the threshold value influences the average number of DPC updates made by one data point during one experiment. The figure confirms that in any graph the TU policy is more efficient than the DU policy, which is because the latter is quicker to update DPCs after passing a node than the former. Moreover, the higher the threshold value, the larger the difference, as higher thresholds mean that the TU policy keeps data points longer at their current edges' nodes before transiting to new edges, while the DU policy updates DPCs immediately in any case. Note that in the TG the disadvantage of using the DU policy is lower than in the OG, because our 2D-to-graph transformation makes edges significantly longer and, consequently, reduces the number of updates.

Moreover, the figure shows that any update policy works more efficiently in the TG than in the OG. This is because of the significantly reduced number of edges and additional updates after passing a node, introduced by both policies. Moreover, the higher the threshold value, the larger the difference, as updates after passing a node then make up a larger part of all updates. The disadvantage of using the OG is lower with the TU policy than with the DU policy, because for each additional edge, the former policy is quicker to update DPCs than the latter.

In summary, the experiments showed that the TG and the TU update policy generally perform better than their alternatives.

\section{CONCLUSIONS}

In this paper, we have proposed a solution to the problem of answering active, ordered $k$-NN queries for moving query and data points in road networks. The paper's contribution is at two levels. First, a general, abstract framework for this and similar problems, including a generic conceptual data model and definitions of relevant abstract functionality, is proposed. Second, a concrete set of algorithms for NN search and a prototype implementation of the data model and algorithms is developed. Initial experiments with the prototype are reported that indicate that the system design, e.g., the use of a special, transformed graph representation for NN query processing and the use of threshold-based updating of data points on the server, results in improved performance.

Possible research directions include the study of variants of active, ordered $k$-NN queries. In particular, it is interesting to consider queries with pre-defined routes, probabilistic queries, and queries in graphs with dynamic weights. Such studies would result in extensions of our data model, system architecture, and algorithms. Additionally, the 2D model and the 2D-to-graph mapping might be extended to capture directional attributes, turn restrictions, etc. Further experiments should also be conducted to study the running time and the scalability (in the number of points) of the algorithms. It would also be of interest to implement and experiment with a prototype that runs in a real environment with mobile users and real-world data.

Acknowledgments. This work was supported in part by grants 216 and 333 from the Danish National Centre for IT Research, by a grant from the Electronics and Telecommunications Research Institute, South Korea, and through contract number IST-2001-32645 from the European Commission.

\section{REFERENCES}

[1] J. Añez, T. de la Barra, and B. Pérez. Dual Graph Representation of Transport Networks. Transportation Research 30(3):209-216, 1996.

[2] R. Benetis, C. S. Jensen, G. Karčiauskas, and S. Šaltenis. Nearest Neighbor and Reverse Nearest Neighbor Queries for Moving Objects. In Proc. IDEAS, pp. 44-53, 2002.

[3] T. Brinkhoff. A Framework for Generating Network-Based Moving Objects. Geoinformatica 6(2):153-180, 2002.

[4] K. L. Cheung and A. W. Fu. Enhanced Nearest Neighbour Search on the R-tree. SIGMOD Record 27(3):16-21, 1998.

[5] H. D. Chon, D. Agrawal, and A. El Abbadi. FATES: Finding A Time dEpendent Shortest path. In Proc. MDM, pp. 165-180, 2003.

[6] E. W. Dijkstra. A Note on Two Problems in Connexion with Graphs. Numerische Mathematik 1:269-271, 1959.

[7] R. H. Güting et al. A Foundation for Representing and Querying Moving Objects. ACM TODS 25(1):1-42, 2000.

[8] K. Johnston, J. M. Ver Hoef, and K. Krivoruchko. Using ArcGIS Geostatistical Analyst. ESRI Press, 316 pp., 2001.

[9] D. E. Knuth. Art of Computer Programming, Volume 3: Sorting and Searching. Addison-Wesley Pub Co, 780 pp., 1998.

[10] C. Murray. Oracle Spatial User Guide and Reference, Release 9.2. Oracle Corporation, 486 pp., 2002.

[11] D. Papadias, J. Zhang, N. Mamoulis, and Y. Tao. Query Processing in Spatial Network Databases. In Proc. VLDB, 2003.

[12] C. Shahabi, M. R. Kolahdouzan, and M. Sharifzadeh. A Road Network Embedding Technique for K-Nearest Neighbor Search in Moving Object Databases. In Proc. ACM GIS, pp. 94-100, 2002.

[13] Z. Song and N. Roussopoulos. K-Nearest Neighbor Search for Moving Query Point. In Proc. SSTD, pp. 79-96, 2001.

[14] Y. Tao, D. Papadias, and Q. Shen. Continuous Nearest Neighbor Search. In Proc. VLDB, pp. 287-298, 2002.

[15] O. Wolfson et al. Databases for Tracking Mobile Units in Real Time. In Proc. ICDT, pp. 169-186, 1999.

[16] B. Zheng, W.-C. Lee, and D. L. Lee. Search K Nearest Neighbors on Air. In Proc. MDM, pp. 181-195, 2003. 